\title{
A Focus on an Atypical Acute Disseminated Encephalomyelitis
}

\author{
Diatewa JE',2,3*, Mpandzou GA ${ }^{2,3}$, Boubayi Motoula-Latou DA2,3, \\ Sounga-Bandzouzi EP2,3, Obonzo Aloba $\mathrm{K}^{3}$ and Ossou-Nguiet PM2,3 \\ 1Unit of Neurology, Department of Medicine, Makélékélé Hospital, Congo \\ ${ }^{2}$ Faculty of Health Sciences, Marien NGOUABI University, Congo \\ ${ }^{3}$ Department of Neurology, University Hospital of Brazzaville, Congo
}

\section{Case Report \\ Volume 4 Issue 1}

Received Date: February 18, 2019

Published Date: March 14, 2019

DOI: $10.23880 /$ nnoaj-16000132

*Corresponding author: Diatewa Josué Euberma, Unit of Neurology, Department of Medicine, Makélékélé Hospital, Department of Neurology, University Hospital of Brazzaville, Faculty of Health Sciences, Marien NGOUABI University, Brazzaville, Congo, BP 15117, Tel: 00242 050951699; E-mail: lejd01@gmail.com

\section{Abstract}

Acute Disseminated Encephalomyelitis (ADEM) is a multifocal demyelinating inflammatory disease. It mainly affects the white matter of the central nervous system. It occurs after an infection or vaccination. In sub-Saharan Africa, its data among adults are almost non-existent in literature. We report the first case of atypical post-infectious ADEM, followed-up from December 2017 to December 2018 at neurology unit of medicine department of Makélékélé hospital in Brazzaville (Congo). ADEM is atypical because of the following peculiarities: diagnostic (medullary lesion which is exclusively cervical and associated with Guillain-Barré syndrome), therapeutic (therapy associating cyclophosphamide with corticoids) and outcome (monophasic outcome influenced by a concomitant lesion of the peripheral nervous system; outcome favourable over 10 months).

Keywords: Acute Disseminated Encephalomyelitis; Guillain-Barré Syndrome; Multiple Sclerosis; Brazzaville

Abbreviations: ADEM: Acute Disseminated Encephalomyelitis; CNS: Central Nervous System; PNS: Peripheral Nervous System; MS: Multiple Sclerosis; EDSS: Expanded Disability Status Scale; MRI: Magnetic Resonance Imaging; CSF: Cerebrospinal Fluid; ENMG: Electroneuromyogram; GBS: Guillain-Barré Syndrome; PRN: Polyradiculoneuropathy; IPS: Internal Popliteal Sciatic; EPS: External Popliteal Sciatic; NO: Not Obtained.

\section{Introduction}

Acute Disseminated Encephalomyelitis (ADEM) is an inflammatory demyelinating disease characterized by diffuse and multifocal white matter lesion of Central
Nervous System (CNS) [1]. The concomitant association of Peripheral Nervous System (PNS) lesion with ADEM, though rare, remains possible [2,3]. ADEM is a rare and particular pathology among adults because of its diagnosis which is close to that of fulminant form (Marbug form) of Multiple Sclerosis (MS), clinical outcome often monophasic, uncodified treatment. The latter depends on sign and symptom severity. Nevertheless, therapy is based on high doses of intravenous corticosteroids associated with immunoglobulins [4-6]. In sub-Saharan Africa, data related to ADEM among adults are almost non-existent in literature [7]. We report the first atypical clinical case of post-infectious monophasic ADEM in a young Congolese adult followed-up in Brazzaville (Congo). 


\section{Neurology \& Neurotherapy Open Access Journal}

\section{Case Report}

A 44-year-old man with no specific history was followed-up from December 2017 to December 2018 at neurology unit of medicine department of Makélékélé Hospital in Brazzaville (Congo). After an episode of nonspecific respiratory infection over the next two weeks, the patient suffered from bilateral and symmetrical sensitivomotor impairment with progressive worsening in three weeks and upward. This impairment was preceded by permanent headaches and helmet. One week before hospital admission, the patient had developed retention of urine that required the establishment of indwelling catheter. He had neither fever nor sign of intracranial hypertension.

On admission, the patient presented bilateral blurred vision, horizontal binocular diplopia and dilation of right pupil with adduction deficit. The fundus oculi and rest of his ophthalmologic examination were normal. He had an obnubilation with Glasgow score of $13 / 15$, flabby and proximo-distal quadriplegia predominant in lower limbs (driving force quoted $1 / 5$ versus $3 / 5$ in upper limbs). He had no meningeal syndrome. Plantar skin reflex was bilaterally indifferent. Multimodal hypoesthesia was found without detecting sensory level. Expanded Disability Status Scale (EDSS) was ranged from 7 to 9. Standard chest X-ray was normal.

Cerebro-medullary Magnetic Resonance Imaging (MRI) (T2-weighted sequences and FLAIR only) was performed because of sign presence of encephalic lesion associated with sphincter disorders. In the encephalic stage, multiple areas of hyper signals measuring more than $2 \mathrm{~cm}$ were found inside the caudate nucleus, striatum and periventricular region. These hyper signals had the same age and were poorly defined, disseminated, asymmetrical (larger to right) and evenly enhanced by gadolinium (Figure 1). It was also noted a hypersignal on the right optic nerve, suggestive of retrobulbar optic neuritis. In the medullary stage, there was an intramedullary hypersignal with fuzzy boundaries, extended over all cervical regions and enhanced by gadolinium, with edema widening the medullary caliber (Figure 2).

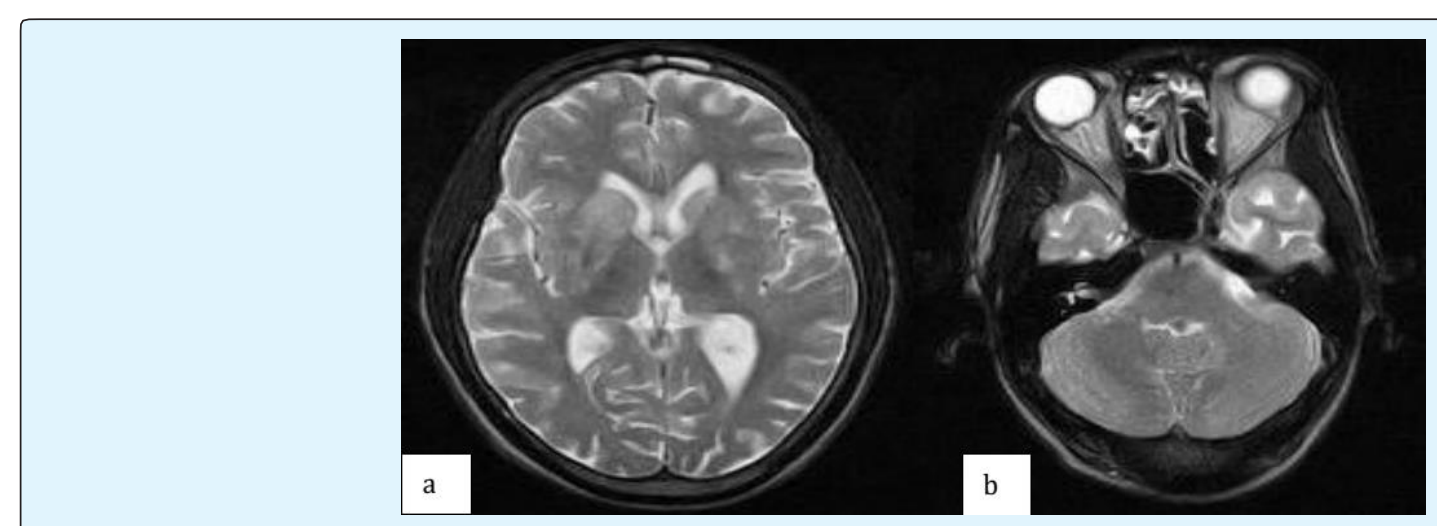

Figure 1: Axial section of brain MRI with gadolinium injection in T2-weighted sequence showing multiple hyper signals affecting gray matter (a) and right optic nerve (b).

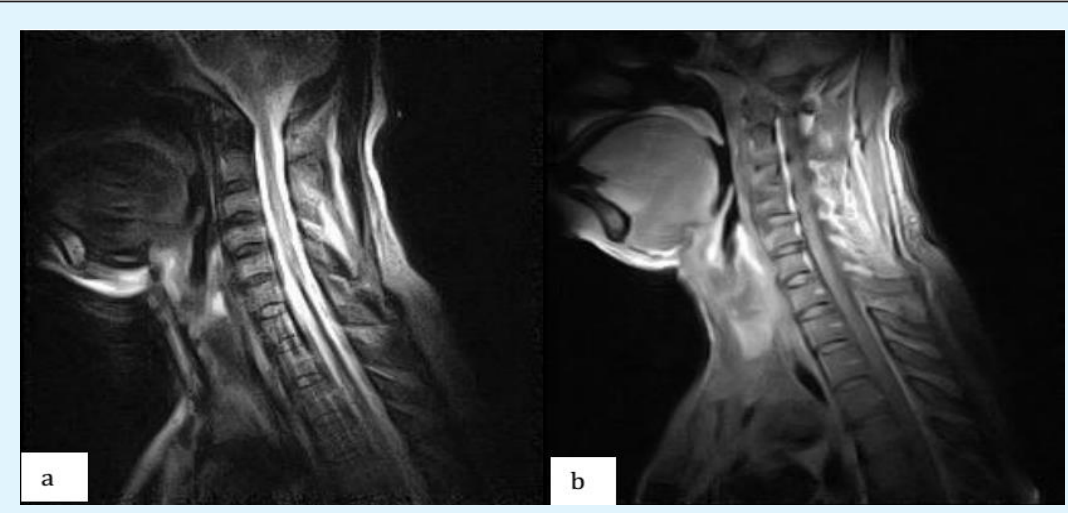

Figure 2: Sagittal section of cervical medullary MRI with gadolinium injection in FLAIR (a) and T2-weighted (b) sequences showing extensive intramedullary hypersignal. 


\section{Neurology \& Neurotherapy Open Access Journal}

On blood examination, it was noted: normochromic normocytic anaemia without leukocytosis (haemoglobin level $=10.5 \mathrm{~g} / \mathrm{dl}$ ); accelerated erythrocyte sedimentation rate $(85 \mathrm{~mm}$ at the first hour) and elevated concentration of C-reactive protein (48 $\mathrm{mg} / \mathrm{l})$, indicating an inflammatory syndrome; normal plasma activity levels of aspartate aminotransferase and alanine aminotransferase; normal plasma concentrations of glucose, urea and creatinine; normal electrophoretogram of serum proteins; negative serological evaluations of human immunodeficiency virus and hepatitis B and C; negative serum testing for anti-aquaporin-4 and anti-myelinoligodendrocyte glycoprotein antibodies.

Cerebrospinal Fluid (CSF) examination showed a clear appearance, mild protein elevation $(0.52 \mathrm{~g} / \mathrm{l})$, normal glucose, white blood cell count of $22 / \mathrm{mm}^{3}$ with 50 percent lymphocytes and negative oligoclonal bands.
Fungal and bacterial cultures were negative. Polymerase chain reactions for Epstein-Barr virus, varicella zoster virus, cytomegalovirus, herpes simplex virus and chlamydia were not performed because of the patient's limited financial resources.

From the motor level, Electroneuromyogram (ENMG) showed a diffuse and homogeneous slowing down of motor conduction velocity, extension of motor distal latency and absence of $\mathrm{F}$ waves in all four limbs, without conduction blocks (Table 1). From the sensory level, potential was not obtained. ENMG revealed a neurogenic pattern; it was characterized by a poor, accelerated pattern with giant potentials on the left anterior tibialis muscle $(10 \mathrm{mV})$ and short abductor muscle of the left thumb $(2.3 \mathrm{mV})$ with no potential signs of denervation or reinnervation at rest (Figure 3). ENMG data showed evidence of Guillain-Barré Syndrome (GBS).

\begin{tabular}{|c|c|c|c|c|c|}
\hline Nerve & Distal Latency (ms) & Amplitude (mV) & Area $(\mu V s)$ & Distance (cm) & Conduction Velocity $(\mathrm{m} / \mathrm{s})$ \\
\hline \multicolumn{6}{|c|}{ Left: Median } \\
\hline Wrist & 8.2 & 2.1 & 6.4 & - & - \\
\hline Elbow & 21 & 2.1 & 12.1 & 29 & 22.7 \\
\hline \multicolumn{6}{|c|}{ Left: Ulnar } \\
\hline Wrist & 5.6 & 2 & 8.2 & - & - \\
\hline Under-elbow & 16.9 & 2.5 & 14 & 25 & 22.1 \\
\hline Above-elbow & 22.9 & 2 & 14.2 & 10 & 16.7 \\
\hline \multicolumn{6}{|c|}{ Right: Ulnar } \\
\hline Wrist & 7 & 2.1 & 10.4 & - & - \\
\hline Under-elbow & 15.3 & 2.1 & 11.4 & 24 & 28.9 \\
\hline Above-elbow & 24.3 & 1.5 & 9.3 & 10 & 11.1 \\
\hline \multicolumn{6}{|c|}{ Right: Median } \\
\hline Wrist & 9.9 & 5 & 22.8 & - & - \\
\hline Elbow & 20.3 & 4 & 22.8 & 29 & 27.9 \\
\hline \multicolumn{6}{|c|}{ Left: IPS } \\
\hline Ankle & NO & NO & NO & - & - \\
\hline Popliteal & NO & NO & NO & - & - \\
\hline \multicolumn{6}{|c|}{ Left: EPS } \\
\hline Ankle & NO & NO & NO & - & - \\
\hline Peroneal & NO & NO & NO & - & - \\
\hline \multicolumn{6}{|c|}{ Right: IPS } \\
\hline Ankle & 9.4 & 0.6 & - & - & - \\
\hline Popliteal & 24.5 & 0.4 & - & 47 & 31.1 \\
\hline \multicolumn{6}{|c|}{ Right: EPS } \\
\hline Ankle & $\mathrm{NO}$ & $\mathrm{NO}$ & $\mathrm{NO}$ & - & - \\
\hline Peroneal & NO & NO & NO & - & - \\
\hline
\end{tabular}

IPS: Internal Popliteal Sciatic; EPS: External Popliteal Sciatic; NO: Not Obtained.

Table 1: Motor conduction studies. 


\section{Neurology \& Neurotherapy Open Access Journal}

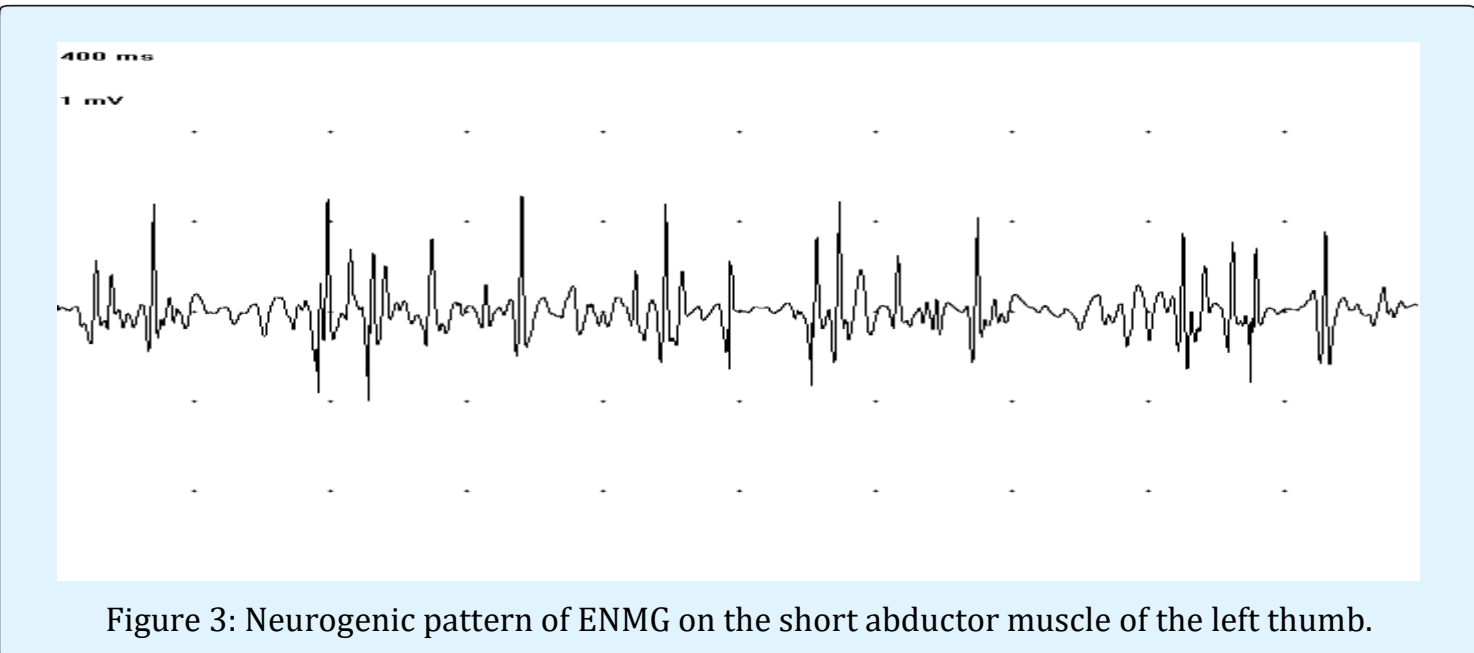

By the light of laboratory, neuroimaging and clinical features, and using investigational evidence of ADEM (Table 2) resulting from criteria reported in literature $[4,8,9]$, we concluded that the patient suffered from atypical ADEM.

\begin{tabular}{|c|}
\hline Criteria $\mathbf{n}^{\circ} \mathbf{1}$ : atypical symptoms \\
\hline Consciousness disorders \\
\hline Hypersomnia \\
\hline Vomiting \\
\hline Epileptic seizures \\
\hline Cognitive disorders \\
\hline Hemiplegia \\
\hline Tetraplegia \\
\hline Aphasia \\
\hline Bilateral retrobulbar optic neuritis \\
\hline Criteria $\mathbf{n}^{\circ} \mathbf{2}$ : biological data \\
\hline Absence of oligoclonal bands \\
\hline Criteria $\mathbf{n}^{\circ} \mathbf{3}$ : radiological data \\
\hline Cortical involvement \\
\hline Involvement of basal ganglia \\
\hline Criteria $\mathbf{n}^{\circ} \mathbf{4}$ : electrophysiological data \\
\hline Concomitant combination of polyradiculoneuropathy \\
\hline Criteria $\mathbf{n}^{\circ} \mathbf{5}$ : outcome \\
\hline Mainly monophasic with possibility of multiphasic \\
\hline evolution if recurrent or multiphasic ADEM criteria \\
present \\
\hline
\end{tabular}

Table 2: Investigational evidence of ADEM.

In this context, an intravenous treatment using bolus of methylprednisolone ( $1 \mathrm{~g}$ /day for 5 days) was instituted with oral relay (via the nasogastric tube) of prednisolone ( $1 \mathrm{mg} / \mathrm{kg} /$ day for 4 weeks). Consciousness return was observed in 10 days by using corticotherapy. Other neurological disorders were unchanged. The extension of corticosteroid therapy, with progressive regression in 8 weeks, associated with motor physiotherapy, led only to diplopia disappearance. Because of clinical outcome which was unsatisfactory, cyclophosphamide was administered as a bolus of $1000 \mathrm{mg} / \mathrm{m}^{2}$ body area every 3 weeks, at the rate of 3 days per cycle. The patient had received 4 boluses of cyclophosphamide taken orally at the rate of $5 \mathrm{mg} / \mathrm{kg} /$ day for 6 weeks and at regressive doses. Using this therapy, it was noted normalization of cerebro-medullary MRI in 6 months and complete regression of neurological disorders in 10 months (negative EDSS score). Recurrence was not observed after one year of follow-up.

\section{Discussion}

The peculiarities of our study are mainly related to the diagnostic criteria, treatment associating cyclophosphamide with corticosteroids and monophasic outcome influenced by the concomitant lesion of the PNS. An important diagnostic difficulty is the presence of various and non-specific clinical signs noticed in ADEM as well as in MS's fulminant form (Marbug form). The use of investigational evidence of ADEM (Table 2) has allowed confirming the diagnosis of atypical ADEM. However, the presence of 4 criteria over 5 also allows retaining it.

Apart from encephalic involvement, ADEM is characterized by extensive myelitis, mainly in the thoracic level [10]. Cervical involvement found in this study is an exceptional status, although it is already reported in literature, mainly among children [11]. The association of PNS lesion with ADEM is unusual. It is often fortuitous $[2,3,12]$. These findings could be explained by lesion 


\section{Neurology \& Neurotherapy Open Access Journal}

severity of CNS, masking that of PNS [3,12], but also by PNS lesion being not systematically sought. PNS lesion is characterized by an acute, axonal or demyelinating Polyradiculoneuropathy (PRN) $[2,13]$; this is the case in our patient. PRN research should be systematic. PNS lesion associated with MS is also possible. It is distinguished by a subacute or chronic PRN [3]. As a result, acute PRN is a diagnostic criterion of certainty for atypical ADEM.

In the case of the ADEM-GBS association, first-line treatment is open to discussion. A study among adults recommends the use of intravenous immunoglobulin [14]. In other studies, first-line treatment is chosen according to the first signs or symptom severity. Corticosteroids and immunoglobulins are used in the treatment of ADEM and peripheral disorders, respectively [2]. In this study, we have used first-line corticosteroid therapy because of the severity of the central lesion. Owing to improvement lack of clinical status, cyclophosphamide was instituted and proved to be very effective. Cyclophosphamide could be considered as a therapeutic alternative in the absence of efficacy of corticosteroids in ADEM [15], but also in GBS [2]. The lack of intravenous immunoglobulin in our workplace has led us to use cyclophosphamide which is recommended as a second-line treatment for acute PRN [2].

Nearly $70 \%$ of patients with ADEM are able to walk without help in 7 months [8]. In our patient, recovery was complete over 10 months. This slow recovery could be explained by the severe peripheral lesion associated, with signs of secondary axonal damage [3,9]. Several predictive factors of recurrence are noted after a first episode of ADEM. Among these factors, there are the existence of an atypical sign of MS (vigilance disorder), absence of CSF oligoclonal bands, existence of optic neuropathy, deep lesion of gray substance revealed by MRI, lesion-associated with GBS, no sequelae after ADEM $[3,4]$. The presence of two predictive factors in patient has a positive predictive value of $97 \%$. Many predictive factors are identified in our patient, indicating that he has a high risk of recurrence. The absence of a new episode 3 months after symptom onset and 4 weeks after immunomodulatory therapy interruption, suggests that, in our patient, recurrence would be unlikely [8]. However, monitoring over an average duration of 38 months remains the best indicator [8].

\section{Conclusion}

ADEM is a rare neurological pathology with variable prognosis. Its clinical fulminant and nonspecific presentation may mimic that of MS's fulminant form (Marbug form). The association of ADEM with GBS is possible and testifies to its gravity. This association must be sought by means of clinical examination and ENMG performing. In this context, in the event of absence of intravenous immunoglobulins, immunosuppressants would be used as soon as possible owing to inefficiency of corticosteroid therapy.

\section{Declaration of Interest}

The authors declare that they have no conflict of interest in relation to this article.

\section{References}

1. Leake JA, Albani S, Kao AS, Senac MO, Billman GF, et al. (2004) Acute disseminated encephalomyelitis in childhood: epidemiologic, clinical and laboratory features. Pediatr infect Diseases 23(8): 756-764.

2. Adamovic T, Riou EM, Bernard G, Vanasse M, Decarie JC, et al. (2008) Acute combined central and peripheral nervous system demyelination in children. Pediatr Neurol 39(5): 307-316.

3. Ogata H, Matsuse D, Yamasaki R, Kawamura N, Matsushita T, et al. (2016) A nationwide survey of combined central and peripheral demyelination in Japan. J Neurol Neurosurg Psychiatry 87(1): 29-36.

4. De Seze J, Debouverie M, Zephir H, Lebrun C, Blanc F, et al. (2007) Acute fulminant demyelinating disease: a descriptive study of 60 patients. Arch Neurol 64(10): 1426-1432.

5. Pellegrino P, Radice S, Clementi E (2014) Geoepidemiology of acute disseminated encephalomyelitis. Epidemiology 25(6): 928-929.

6. Yong $\mathrm{C}$, Fubao $\mathrm{M}$, Yuanling $\mathrm{X}$, Xuhua Chu, Jinlin $\mathrm{Z}$ (2015) Incidence of acute disseminated encephalomyelitis in the Jiangsu province of China, 2008-2011. Mult Scler J Exp Transl Clinical 1: 1-7.

7. Howlett WP (2014) Inflammatory neurologic disease in sub-Saharan Africa. Neurology 83(7): 656-658.

8. Tenembaum S, Chitnis T, Ness J, Hahn JS (2007) Acute disseminated encephalomyelitis. Neurology 68(16): 23-36.

9. Berzero G, Cortese A, Ravaglia S, Marchioni E (2016) Diagnosis and therapy of acute disseminated 


\section{Neurology \& Neurotherapy Open Access Journal}

encephalomyelitis and its variants. Expert Review of Neurotherapeutics 16(1): 83-101.

10. Dale RC (2003) Acute disseminated encephalomyelitis. Semin Pediatr Infect Dis 14(2): 90-95.

11. Elbouderkaoui M, Ajdakar S, Rada N, Draiss G, Bouskraoui M (2015) Association d'une encéphalomyélite aiguë disséminée et syndrome de Guillain-Barré chez l'enfant. Pan African Medical Journal $21: 184$.

12. Marchioni E, Ravaglia S, Piccolo G, Furione M, Zardini E, et al. (2005) Postinfectious inflammatory disorders
: Subgroups based on prospective follow-up. Neurology 65(7): 1057-1065.

13. Marchioni E, Ravaglia S, Montomoli C, Tavazzi E, Minoli L, et al. (2013) Postinfectious neurologic syndromes: a prospective cohort study. Neurology 80(10): 882-889.

14. Yuki N (2009) Fisher syndrome and Bickerstaff brainstem encephalitis (Fisher-Bickerstaff syndrome). J Neuroimmunol 215(1-2): 1-9.

15. De Seze J (2010) Encéphalomyélites aiguës disséminées. Neurology.com 2(7): 176-178. 